\title{
A influência da modernidade no comportamento alimentar: Uma revisão
}

\author{
The influence of modernity on eating behavior: A review \\ La influencia de la modernidad em la conducta alimentaria: Una revisión
}

Recebido: 14/03/2021 | Revisado: 29/03/2021 | Aceito: 20/04/2021 | Publicado: 09/05/2021

\author{
Martha Teresa Siqueira Marques Melo \\ ORCID: https://orcid.org/0000-0002-5858-9298 \\ Universidade Federal do Piaú, Brasil \\ E-mail:marthateresa.melo@ufpi.edu.br \\ Ana Cláudia Carvalho Moura \\ ORCID: https://orcid.org/0000-0002-9930-8552 \\ Universidade Federal do Piauí, Brasil \\ E-mail: anaclaudiacarvalho@ufpi.edu.br \\ Gleyson Moura dos Santos \\ ORCID: https://orcid.org/0000-0003-0904-1995 \\ Universidade Federal do Piauí, Brasil \\ E-mail:g_leyson_moura@hotmail.com \\ Bianca Lourrany dos Santos Silva \\ ORCID: https://orcid.org/0000-0001-9432-5268 \\ Universidade Federal do Piaú, Brasil \\ E-mail:biancalourrany@ufpi.edu.br \\ Beatriz Bianca Miranda Silva \\ ORCID: https://orcid.org/0000-0001-7182-6377 \\ Universidade Federal do Piauí, Brasil \\ E-mail: bia.miranda.2303@gmail.com \\ Ivone Freires de Oliveira Costa Nunes \\ ORCID: https://orcid.org/0000-0002-9890-1231 \\ Universidade Federal do Piauí, Brasil \\ E-mail: ivonefreirescosta@ufpi.edu.br \\ Ana Raquel Soares Oliveira \\ ORCID: https://orcid.org/0000-0001-5383-0137 \\ Universidade Federal do Piauí, Brasil \\ E-mail:ana-raqueloliveira@hotmail.com \\ Luana Mota Martins \\ ORCID: https://orcid.org/0000-0002-2521-5820 \\ Centro Universitário Maurício de Nassau, Brasil \\ E-mail:lua_mota@hotmail.com \\ Suely Carvalho Santiago Barreto \\ ORCID: https://orcid.org/0000-0001-7426-5173 \\ Universidade Federal do Piauí, Brasil \\ E-mail:csantiago.suely@gmail.com \\ Lila Cristina Xavier Luz \\ ORCID: https://orcid.org/0000-0001-7301-0187 \\ Universidade Federal do Piauí, Brasil \\ E-mail: lilaxavier@hotmail.com \\ Paulo Víctor de Lima Sousa \\ ORCID: https://orcid.org/0000-0003-1486-0661 \\ Centro Universitário Maurício de Nassau, Brasil \\ E-mail: paulovictor.lima@hotmail.com \\ Cecilia Maria Resende Gonçalves de Carvalho \\ ORCID: https://orcid.org/0000-0002-8707-1447 \\ Universidade Federal do Piauí, Brasil \\ E-mail:ceciliacvalho@ufpi.edu.br
}

\begin{abstract}
Resumo
O objetivo deste estudo foi verificar as modificações do comportamento alimentar influenciadas pela vida moderna. Trata-se de uma revisão integrativa da literatura realizada nas bases de dados Publisher Medline (PubMed), Science Direct e Web of Science com descritores pré-selecionados. Os resultados apontaram que a alimentação, comensalidade e cultura alimentar sofrem interferência diante da vida moderna e da globalização. Observou-se mudanças drásticas nos hábitos e práticas alimentares, que podem contribuir para a redução da qualidade da alimentação, diminuição da socialização e compartilhamento de refeições entre familiares, difusão e fragmentação de culturas alimentares. O ato de comer com prazer pode ser comprometido, pela pressa da rotina diária, distância de casa, ausência de interação
\end{abstract}


social ou por falta de opções de alimentos no mercado com os quais os indivíduos se identifiquem próprios da sua cultura. Ademais, existe uma inclinação à supervalorização da cultura alimentar de origem e a tentativa de sua reprodução, principalmente quando se convive com culturas diferentes, dentro das quais a culinária pode ser um agente de exclusão social, sendo o resgate da cultura de origem uma maneira de se reafirmar como indivíduo. Nesse contexto, as perspectivas são preocupantes, pois se espera uma diminuição ainda maior na interação social durante as refeições.

Palavras-chave: Comportamento alimentar; Dieta; Cultura.

\begin{abstract}
The aim of this study was to verify changes in eating behavior influenced by modern life. This is an integrative literature review carried out in the Publisher Medline (PubMed), Science Direct and Web of Science databases with pre-selected descriptors. The results showed that food, commensality and food culture suffer interference in the face of modern life and globalization. There were drastic changes in eating habits and practices, with a tendency to worsen the quality of meals, decreased socialization and sharing of meals among family members, diffusion and fragmentation of food cultures. The act of eating with pleasure can be compromised, due to the rush of daily routine, distance from home, absence of social interaction or lack of food options in the market with which individuals identify themselves with their culture. Furthermore, there is an inclination towards overvaluing the food culture of origin and the attempt to reproduce it, especially when living with different cultures, within which cuisine can be an agent of social exclusion, with the rescue of the culture of origin being a way of reaffirm yourself as an individual. In this context, the prospects are worrying, as an even greater decrease in social interaction during meals is expected.
\end{abstract}

Keywords: Feeding behavior; Diet; Culture.

\title{
Resumen
}

El objetivo de este estudio fue verificar los cambios en la conducta alimentaria influenciados por la vida moderna. Se trata de una revisión integradora de la literatura realizada en las bases de datos Publisher Medline (PubMed), Science Direct y Web of Science con descriptores preseleccionados. Los resultados mostraron que la alimentación, la comensalidad y la cultura alimentaria sufren interferencias frente a la vida moderna y la globalización. Hubo cambios drásticos en los hábitos y prácticas alimentarias, con tendencia a empeorar la calidad de las comidas, disminución de la socialización y distribución de comidas entre los miembros de la familia, difusión y fragmentación de las culturas alimentarias. El acto de comer con placer puede verse comprometido, por el apuro de la rutina diaria, la lejanía del hogar, la ausencia de interacción social o la falta de opciones de comida en el mercado con las que los individuos se identifiquen con su cultura. Además, existe una inclinación a sobrevalorar la cultura alimentaria de origen y el intento de reproducirla, especialmente cuando se convive con culturas diferentes, dentro de las cuales la cocina puede ser un agente de exclusión social, siendo el rescate de la cultura de origen una forma de reafirmese como individuo. En este contexto, las perspectivas son preocupantes, ya que se espera una disminución aún mayor de la interacción social durante las comidas.

Palabras clave: Conducta alimentaria; Dieta; Cultura.

\section{Introdução}

A alimentação não se situa somente no âmbito fisiológico, mas assume um comportamento cultural (Lima, Ferreira Neto \& Farias, 2015). A fome biológica distingue-se dos apetites, anseios humanos e contentamento proporcionado pelo ato de levar o alimento do prato à boca, pois se consolida em atitudes, hábitos, costumes, rituais e etiquetas (Carneiro, 2017).

Nesse contexto, atitudes alimentares envolvem crenças, pensamentos, sentimentos e comportamento com os alimentos. O comportamento alimentar abrange um conjunto de cognições e afetos que conduzem as ações alimentares, como o modo de comer. Esse comportamento quando frequente torna-se um hábito alimentar, o costume e modo de comer relacionado à pessoa (Alvarenga, Figueiredo, Timerman \& Antonaccio, 2019).

O comportamento alimentar está interligado com o ato de comer, cultura, sociedade e experiência com o alimento (Alvarenga et al., 2019). O ato de comer em companhia, com familiares e pessoas de seu convívio, proporciona mais prazer e valorização da alimentação (Santos \& Sousa, 2021), pode contribuir para o consumo adequado de alimentos e interação social. Além da comensalidade, torna-se importante a adoção de práticas que favoreçam o comer devagar, com regularidade, atenção e o desfrutar do que se come; comer em ambiente apropriado e compartilhar atividades domésticas relacionadas às refeições (Brasil, 2014). 
A alimentação é um tema abrangente para explorar os múltiplos significados da globalização, uma vez que expõe a complexidade desse fenômeno peculiar que transcende o aspecto econômico. Embora existam relações com a cultura local, a religião, o gosto, a tradição, o simbolismo e a identidade, a comida tem sido produzida como uma mercadoria sob as premissas de um sistema e de uma política agroalimentar de caráter global, dominada por corporações agroalimentares transnacionais, o que envolve uma forma legitimada de agrobiopoder e de ameaça à soberania alimentar, além de impactos culturais, socioeconômicos e ambientais significativos (Azevedo, 2017).

As modificações constantes e aceleradas no sistema de produção e processamento de alimentos, bem como a globalização das economias, costumes e mercados, inserem as práticas alimentares em um ambiente mutável, uma vez que na complexa sociedade urbano-industrial os processos de transformação são muito rápidos, fazendo com que o indivíduo os reproduza socialmente de forma fragmentada em seu próprio meio. As tradições culinárias se confrontam com a transição, adaptação e ressignificação de ingredientes, utensílios e saberes. A literatura acerca das temáticas de sociologia e antropologia da alimentação revela que as cozinhas e culinárias de grupos sociais são espaços de observação de culturas que estão em constante mudança (Zaneti, 2016).

A expansão da indústria e do mercado de alimentação fora do lar são alterações significativas, promovidas não apenas por avanços tecnológicos na forma de produzir, armazenar, preparar e servir alimentos, mas também por questões de caráter socioeconômico e cultural, bem como mudanças no estilo de vida das pessoas (Luizetto, Tureck, Locatelly, Correa \& Koehnlein, 2015).

As mudanças socioculturais atingiram a rotina familiar e todos os elementos presentes neste contexto, como os hábitos e comportamentos referentes à alimentação. As famílias muitas vezes não conseguem compartilhar refeições, o que pode estar relacionado aos compromissos profissionais e pessoais, a inserção maciça de mulheres ao mercado de trabalho, às distâncias nos grandes centros urbanos e dificuldades no trânsito. Diante dessa nova realidade, as famílias precisaram se reorganizar, o que implica consequências emocionais, comportamentais e nutricionais ainda não mensuradas (Carvalho, Bastos \& GimenesMinasse, 2017).

Considerando o exposto, objetivou-se realizar um estudo de revisão integrativa da literatura a respeito das modificações do comportamento alimentar influenciadas pela vida moderna.

\section{Metodologia}

Trata-se de um estudo de revisão integrativa da literatura que compreendeu as seguintes etapas: definição do tema (Comportamento alimentar na modernidade) e da questão norteadora (Qual a influência da vida moderna nas modificações do comportamento alimentar?); estabelecimento dos critérios de elegibilidade (artigos originais com a temática, publicados em inglês, no período de 2014 a 2020, realizados com indivíduos sadios, de ambos os sexos, com idade a partir de 18 anos); busca das palavras-chave nos descritores de saúde (pesquisa dos artigos, na qual ocorre a identificação dos artigos nas bases de dados); exclusão de artigos duplicados, por título e resumo e, leitura na íntegra dos artigos para extração dos dados da presente pesquisa (objetivos, metodologia, principais achados, conclusões).

O levantamento dos artigos ocorreu em janeiro de 2021 nas bases de dados Publisher Medline (PubMed), Science Direct e Web of Science, por dois autores, de forma independente, com o uso dos descritores feeding behavior, feeding, culture e food consumption, modernization e globalization, bem como utilizou-se os operadores boleanos AND e OR para a combinação destes descritores na busca. Em caso de discordâncias entre os avaliadores, esta foi resolvida em consenso por meio de discussão em grupo com os pesquisadores.

O estudo envolveu artigos originais do tipo transversal, qualitativo-quantitativo publicados no período de 2014 a 2020 que atenderam os critérios de elegibilidade. Em seguida, fez-se a leitura completa do texto e mediante análise crítica, elaborou- 
se um mapa analítico com as principais informações, categorizando-as em subtemas a fim de se responder a questão norteadora.

Não foram incluídos na revisão os artigos que envolviam animais, crianças e adolescentes, indivíduos com distúrbios alimentares, doenças que requeiram um regime alimentar e/ou agravos digestivos ou intestinais. Para a exposição dos resultados, foram coletadas informações relevantes dos artigos elegíveis, incluindo ano e país do estudo, nomes dos autores, título e principais resultados. Os dados foram dispostos em quadros distribuídos de acordo com o país e ano da publicação.

\section{Resultados e Discussão}

A busca nas bases de dados originou 706 artigos. Após o processo de triagem, remoção de artigos duplicados, leitura na íntegra e análise dos critérios de inclusão e exclusão, 10 (dez) artigos foram selecionados nesta revisão para análise dos dados (Quadro 1).

Quadro 1. Etapas para seleção e análise dos artigos.

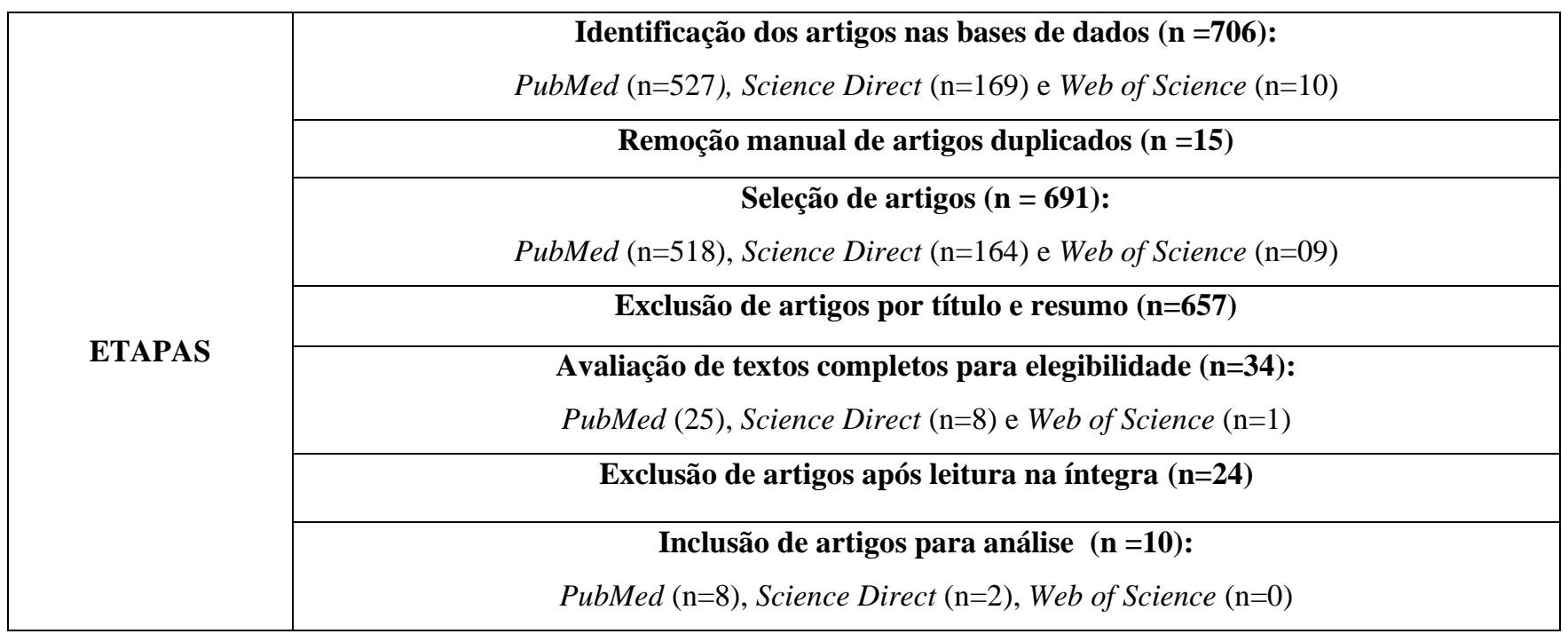

Fonte: Dados da pesquisa (2021). 
Quadro 2. Distribuição dos artigos segundo o autor principal, ano de publicação, país, título, amostra (n), objetivo de desfecho.

\begin{tabular}{|c|c|c|c|c|}
\hline $1^{\circ}$ Autor/Ano/País & Tipo de estudo & $\mathbf{n}$ & Objetivo & Desfecho \\
\hline $\begin{array}{l}\text { Cho/2015/Japão e } \\
\text { Coréia*. }\end{array}$ & $\begin{array}{l}\text { Estudo } \\
\text { Coorte. }\end{array}$ & $\begin{array}{l}\text { Estudo integrado por } 185 \\
\text { estudantes universitários, de } \\
\text { ambos os sexos, com idade entre } \\
18 \text { e } 27 \text { anos. }\end{array}$ & $\begin{array}{l}\text { Investigar diferentes experiências entre } \\
\text { comensalidade e alimentação individual entre } \\
\text { alunos e variações transculturais destas } \\
\text { experiências entre grupos coreanos e japoneses. }\end{array}$ & $\begin{array}{l}\text { Em um ambiente não ocidental (Japão e Coreia) ocorreram } \\
\text { variações transculturais de percepções e práticas de comensalidade } \\
\text { e alimentação individual. }\end{array}$ \\
\hline $\begin{array}{l}\text { Hooper/2015/Nova } \\
\text { Zelândia**. }\end{array}$ & $\begin{array}{ll}\text { Transversal } & - \\
\text { qualitativo. } & \end{array}$ & $\begin{array}{l}\text { Estudo composto por } 16 \text { mulheres } \\
\text { com idade de } 23 \text { a } 64 \text { anos, com } \\
\text { mediana de } 49 \text { anos. }\end{array}$ & $\begin{array}{l}\text { Estudar a associação entre a sincronização das } \\
\text { refeições e a comensalidade através de uma } \\
\text { análise comparativa de dados de pesquisas em } \\
\text { Santiago e Paris. }\end{array}$ & $\begin{array}{l}\text { As experiências alimentares da infância são transportadas para a } \\
\text { fase adulta, influenciando as percepções e preferências sobre o } \\
\text { domínio alimentar. }\end{array}$ \\
\hline Roche/2015/ EUA*. & Exploratório. & $\begin{array}{l}\text { Pesquisa integrada por } 52 \text { mães e } \\
\text { pais de jovens hmong, latinos e } \\
\text { brancos. }\end{array}$ & $\begin{array}{l}\text { Examinar os capitais sociais e culturais } \\
\text { percebidos associados às práticas alimentares } \\
\text { familiares entre mães e pais hmong, latinos e } \\
\text { brancos. }\end{array}$ & $\begin{array}{l}\text { Identificação e promoção do vínculo e ponte de ativos culturais } \\
\text { para práticas alimentares saudáveis entre diferentes grupos } \\
\text { culturais, que podem ser importantes para o desenvolvimento de } \\
\text { programas de educação nutricional entre etnias na comunidade. }\end{array}$ \\
\hline $\begin{array}{l}\text { Giacoman/2016/ } \\
\text { Chile*. }\end{array}$ & $\begin{array}{l}\text { Transversal - } \\
\text { qualitativo. }\end{array}$ & $\begin{array}{l}\text { Estudo composto por } 89 \text { adultos, } \\
\text { de ambos os sexos. }\end{array}$ & $\begin{array}{l}\text { Examinar a importância da alimentação } \\
\text { comunitária entre adultos de Santiago, Chile, } \\
\text { elaborando um modelo teórico para a } \\
\text { comensalidade baseado em material empírico. }\end{array}$ & $\begin{array}{l}\text { A comensalidade fortalece a coesão entre os membros de um } \\
\text { grupo, proporcionando um espaço interativo no qual o } \\
\text { pertencimento é simbolizado e as normas compartilhadas são } \\
\text { respeitadas. Por outro lado, pode ser percebida como negativa, } \\
\text { dependendo do grupo e do contexto, pode ocasionar tensões. }\end{array}$ \\
\hline Guillemi/2016/França*. & $\begin{array}{l}\text { Transversal } \\
\text { quantitativo } \\
\text { qualitativo. }\end{array}$ & $\begin{array}{l}\text { Pesquisa constituída por } 198 \\
\text { indivíduos saudáveis, com idade } \\
\geq 18 \text { anos, ambos os sexos e } \\
\text { eutróficos (índice de massa } \\
\text { corporal } \geq 18,5 \mathrm{e} \leq 24,9 \mathrm{~kg} / \mathrm{m}^{2} .\end{array}$ & $\begin{array}{l}\text { Descrever o desenvolvimento e validação } \\
\text { preliminar de um instrumento de avaliação de } \\
\text { bem-estar associados à alimentação e hábitos } \\
\text { alimentares em uma população saudável em } \\
\text { geral. }\end{array}$ & $\begin{array}{l}\text { O Well-BFQ é uma ferramenta inovadora que avalia como a } \\
\text { população saudável em geral descreve o bem-estar em relação aos } \\
\text { seus alimentos e hábitos alimentares. }\end{array}$ \\
\hline $\begin{array}{l}\text { Holm/2016/Dinamarca } \\
*\end{array}$ & $\begin{array}{ll}\text { Transversal } & - \\
\text { quantitativo. } & \end{array}$ & $\begin{array}{l}\text { Utilização de dados de duas } \\
\text { pesquisas com população adulta: } \\
\mathrm{n}=4823 \text { e } \mathrm{n}=8242 \text {. }\end{array}$ & $\begin{array}{l}\text { Analisar as mudanças na conduta de comer nos } \\
\text { países nórdicos ao longo do período } \\
\text { compreendido entre } 1997 \text { e } 2012 \text {. }\end{array}$ & $\begin{array}{l}\text { O local principal para comer dos nórdicos permaneceu sendo em } \\
\text { casa e no local de trabalho; } \\
\text { As seguintes práticas alimentares aumentaram. } \\
\text { - Comer sozinho; } \\
\text { - Comer diante de Telas, principalmente entre os indivíduos mais } \\
\text { jovens. }\end{array}$ \\
\hline Sato/2016/Brasil*. & $\begin{array}{l}\text { Transversal - } \\
\text { quantitativo. }\end{array}$ & $\begin{array}{l}\text { Constituído por } 439 \text { mães, com } \\
\text { idade entre } 18 \text { a } 55 \text { anos, } \\
(33,5 \pm 7,21) \text {. } \\
\text { IMC }=25.91 \mathrm{~kg} / \mathrm{m} 2( \pm 5.29) \text {. }\end{array}$ & $\begin{array}{l}\text { Investigar a frequência de refeições realizadas } \\
\text { com a família entre mães residentes em Santos, } \\
\text { São Paulo - Brasil. }\end{array}$ & $\begin{array}{l}\text { A realização de refeições em família foi maior entre as mães } \\
\text { responsáveis por cozinhar. } \\
\text { Quando havia pouca comida, o compartilhamento de refeições foi } \\
\text { maior entre as mães que consumiam menor quantidade de } \\
\text { alimentos, do que outros membros da família. }\end{array}$ \\
\hline
\end{tabular}




\begin{tabular}{|c|c|c|c|c|}
\hline Scagliusi/2016/Brasil*. & $\begin{array}{l}\text { Transversal } \\
\text { qualitativo. }\end{array}$ & $\begin{array}{l}\text { Estudo integrado por } 30 \text { mães } \\
\text { trabalhadoras de universidades } \\
\text { públicas da baixada santista. }\end{array}$ & $\begin{array}{l}\text { Analisar os discursos de mães trabalhadoras sobre } \\
\text { as refeições familiares realizadas na mesa, no } \\
\text { sofá e na cama / quarto. }\end{array}$ & $\begin{array}{l}\text { A realização de refeições na mesa, foi considerada como momento } \\
\text { agradável para poucas pessoas. } \\
\text { - O sofá foi considerado o local que a família realiza as refeições, } \\
\text { um espaço menos formal e rígido, mas capaz de permitir algum } \\
\text { convívio coletivo. } \\
\text { - Comer na cama/quarto foi a prática menos comum. } \\
\text { - Assistir televisão é uma prática habitual, principalmente nas } \\
\text { refeições feitas no sofá. }\end{array}$ \\
\hline Bailey/2017/Holanda*. & $\begin{array}{l}\text { Transversal } \\
\text { quantitativo } \\
\text { qualitativo. }\end{array}$ & $\begin{array}{l}\text { Amostra constituída por } 30 \\
\text { indivíduos, com idade de } 25 \text { a } 50 \\
\text { anos, ambos os sexos. }\end{array}$ & $\begin{array}{l}\text { Avaliar as experiências dos participantes em } \\
\text { relação aos alimentos, práticas alimentares e } \\
\text { comensalidade para obter uma compreensão mais } \\
\text { profunda das trocas de cuidados entre os } \\
\text { migrantes e suas famílias em casa. }\end{array}$ & $\begin{array}{l}\text { As experiências dos migrantes em relação alimentos e práticas } \\
\text { alimentares que trouxeram na migração, ajudou a comensalidade } \\
\text { em relação: } \\
\text { - Sentimento: comida de casa e suas memórias e pertencimento; } \\
\text { - Sentido de casa: culinária e práticas alimentares; } \\
\text { - Senso de comunidade: comensalidade com co-etnias, com laços } \\
\text { de comunidade mais fortes; } \\
\text { - Senso de "copresença": comida e cuidado. A comensalidade com } \\
\text { grupos não indígenas foi considerada problemática. }\end{array}$ \\
\hline Utter/2018/EUA**. & $\begin{array}{l}\text { Transversal - } \\
\text { analítico. }\end{array}$ & $\begin{array}{l}\text { Estudo composto por um } \mathrm{n}=889 \text {, } \\
\text { com participantes de ambos os } \\
\text { sexos, idade média de } 31 \text { anos. }\end{array}$ & $\begin{array}{l}\text { Ampliar o que já se sabe sobre o relacionamento } \\
\text { entre as refeições em família e a saúde e bem- } \\
\text { estar dos pais. }\end{array}$ & $\begin{array}{l}\text { - Aumento da realização de refeições familiares frequentes } \\
\text { associado ao comportamento, bem-estar social e emocional dos } \\
\text { pais; maior funcionamento familiar; maior relacionamento entre os } \\
\text { participantes e aos níveis mais baixos de sintomas depressivos, } \\
\text { menor estresse. } \\
\text { - O bem-estar emocional dos pais pode tornar difícil para as } \\
\text { famílias comerem juntas e vice-versa. }\end{array}$ \\
\hline
\end{tabular}

Fonte: Dados da pesquisa (2021). 
Do total de artigos incluídos nesta pesquisa ( $\mathrm{n}=10)$, destaca-se a base de dados PubMed com 80,0\% das publicações $(n=8)$. Não houve publicação referente a temática em questão nos anos de 2014, 2019 e 2020, 2016 destacou-se com mais artigos publicados $(n=5 ; 50,0 \%)$. Os estudos foram ocorreram em diversos países. O Brasil $(n=2)$ e os EUA $(n=2)$ foram os países com mais pesquisa. Em relação ao tipo de estudo, predominou estudos transversais, com abordagem quantitativa e/ou qualitativa, realizados com pessoas adultas, saudáveis, de ambos os sexos, mães e pais. Os artigos selecionados investigaram as mudanças do comportamento alimentar em diversos contextos, como as experiências entre a comensalidade e alimentação individual, sincronização das refeições e a comensalidade, conduta de comer, alimentos, práticas alimentares e comensalidade, dentre outras.

No Quadro 2 tem-se a distribuição das publicações segundo o ano, país onde o estudo foi realizado, título e autor. Os estudos foram realizados em diversos países, dentre os quais destacam-se o Brasil e os EUA, com duas publicações cada.

Em referência ao Quadro 2, observa-se que a alimentação se caracteriza por um processo cultural, no qual proporciona uma relação entre seus comensais, no entanto práticas relacionadas ao estilo de vida ocidental faz com que esses hábitos sejam modiicados. Após a leitura crítica dos artigos incluídos na revisão, verificou-se que essas publicações abordaram aspectos concernentes aos subtemas: comensalidade e comer sozinho, domínio alimentar, modernidade e padrões alimentares, refeições realizadas em família, bem como a relação entre alimento, cultura e comensalidade:

Em relação a comensalidade e comer sozinho, analisando os dados de uma pesquisa transversal com estudantes universitários japoneses e coreanos ( $\mathrm{n}=185)$ com idade entre 18 a 27 anos, Cho, Takeda, Oh, Aiba e Lee (2015) evidenciaram que os estudantes coreanos preferem comer acompanhados, pois quando estão sozinhos, tendem a experienciar emoções negativas, a consumir mais alimentos. Já os japoneses demonstram emoções mistas em relação à essa prática de comer só, declararam não ter preferência pela comensalidade e que não havia diferença na quantidade de alimentos consumidos quando estavam com ou sem companhia. A comensalidade e o consumo de alimentos representam práticas da alimentação cotidiana nas sociedades modernas, quase sempre sendo evidenciadas emoções negativas quando indivíduos comem sozinhos, importantes na determinação das experiências alimentares, incluindo sentimentos e ingestão de alimentos associados a elas, que podem influenciar na saúde física e mental a longo prazo. Comer junto ou sozinho pode influenciar nas respostas do sujeito a qualquer questão relacionada à dieta, porque a alimentação é inseparável da sociabilidade cotidiana.

No âmbito do domínio alimentar quatro estudos (Hooper, Ivory \& Fougere, 2015; Roche, Goto, Zhao \& Wolff, 2015; Giacoman, 2016; Guillemi, Marrel, Arnould, Capuron, Dupuy, Ginon, Layé, Lecerf, Prost, Rogeaux, Urdapilleta \& Allaert, 2016) foram analisados. Em 2015, Hooper, Ivory e Fougere realizaram na Nova Zelândia, uma pesquisa com mulheres (n =16), idade de 23 a 64 anos, que avaliou de que modo as preferências e percepções alimentares incorporadas durante a infância influenciam as práticas da vida adulta, saúde. A pesquisa considerou lar e vizinhança como ambientes sociais que determinam as relações sociais e alimentação como fator importante para criação e manutenção de relações sociais. Os dados coletados correspondiam a fatores socioeconômicos, dados antropométricos e percepções e atitudes das participantes em relação ao ambiente construído e sua vizinhança local. Os achados sugerem que o envolvimento alimentar de adultos é influenciado pelas experiências vivenciadas na infância e a construção e reflexão a respeito desse tema recorda essa fase.

As participantes cujo envolvimento alimentar era relativamente baixo ilustraram suas práticas atuais por meio de descrições e reflexões avaliativas com lembranças negativas, desagradáveis e angustiantes da infância. Para esses indivíduos, as refeições da infância são lembradas como campo de batalha e o domínio alimentar, na idade adulta, é um incômodo necessário, sendo prejudicial à sua saúde e bem-estar. Já as participantes com alto envolvimento alimentar avaliaram suas práticas sem gerar um contexto de tensão e angústia ao retomarem a infância, mesmo quando essas lembranças sugerem ideias conflitantes sobre alimentação entre pais e filhos. Essas mulheres refletiam sobre as refeições na infância como um momento agradável e descreviam diversas filosofias alimentares que proporcionavam prazer e alegria (Hooper, Ivory \& Fougere, 2015). 
Na pesquisa qualitativa realizada por Roche et al., (2015), com grupo focal com pais e mães Hmong e latinos (n=52), a valorização dos alimentos tradicionais deu-se no grupo étnico (Hmong), enquanto os latinos eram mais receptíveis a alimentos novos de outras culturas. Os participantes divergiram nas opiniões quanto ao papel do gênero nas práticas familiares, bem como nos esforços para a preservação da cultura alimentar. Esse estudo mostrou que em diferentes grupos culturais a identificação e a promoção do vínculo e a ligação de bens culturais, na perspectiva de práticas alimentares saudáveis, pode ser importante para o desenvolvimento de programas de educação nutricional bem-sucedidos em todas as etnias da comunidade.

Já no estudo qualitativo de Giacoman (2016), realizado no Chile com adultos ( $\mathrm{n}=89$ ), foram feitas 24 entrevistas em grupo. Nelas, os participantes foram selecionados de maneira proposital, escolhendo os grupos de acordo com o tipo de relacionamento (familiar, profissional ou amizade) e tipo de comensalidade (ambiente doméstico ou ambiente não-doméstico). As entrevistas realizadas exibiram uma variedade de práticas e valores em relação às refeições compartilhadas, influenciadas pelas características próprias de cada grupo. Porém, em meio às diferenças observadas, todas as narrativas convergem para o mesmo ponto: as refeições são percebidas como uma atividade que une os membros de um grupo e fortalece o sentimento de identidade dentro desse grupo. Desse modo, a comensalidade como prática, desempenha um papel de união entre membros de um grupo, proporcionando um espaço interativo, no qual os indivíduos se sentem representados e as normas compartilhadas são respeitadas. Por outro lado, ao passo que a comensalidade é vista como positiva ao permitir conexões entre membros de um grupo, também pode ocasionar conflitos, dependendo das características do grupo comensal em questão.

O desenvolvimento e validação de um questionário (Well-BFQ) por Guillemi et al., (2016), foram obtidos a partir de dados qualitativos de grupos de discussão com indivíduos saudáveis $(\mathrm{n}=198)$, idade $\geq 18 \mathrm{anos}$, ambos os sexos. Diversos temas foram discutidos, dentre os quais os tipos de alimentos e hábitos alimentares, compras e preparação de alimentos (utensílios, modo de cozimento, ingredientes), hábitos alimentares gerais, contexto e ambiente da refeição (sozinho, em companhia, ambiente calmo, barulhento). Essa discussão possibilitou abranger o domínio de bem-estar relacionado ao prazer e ao alimento e o domínio de bem-estar relacionado à alimentação e à saúde.

No domínio de bem-estar relacionando prazer ao alimento, as citações dos participantes referem-se à importância de sair da rotina diária para realizar compras, comer em companhia; descoberta de novos pratos e novos sabores e redescoberta de pratos antigos; comida; prazer em comer; prazer visual; utilização de produtos locais e de boa procedência; cozinhar e compartilhar com outras pessoas e outros atributos que dão prazer, que são também importantes para o alcance de uma alimentação saudável. No domínio do bem-estar relacionado com a alimentação e saúde, os participantes destacaram: uso de comida local, para a preservação ecológica; utilização de alimentos orgânicos e alimentos frescos, devido à qualidade nutricional; alimentação variada e balanceada; moderação no consumo alimentar, evitando os alimentos com elevado teor de sal e gordura; as refeições estruturadas em relação ao número e ao horário; não omissão de refeições e não beliscar entre as refeições. Diante dos resultados alcançados, os pesquisadores concluíram que o Well-BFQ é uma ferramenta única e modular que avalia de forma abrangente o quadro completo de bem-estar relacionado à alimentação e hábitos alimentares na população em geral (Guillemi et al., 2016).

$\mathrm{Na}$ presente revisão foram, também, evidenciadas as mudanças ocorridas nos padrões alimentares devido a vida moderna (modernidade e padrões alimentares). Nesse contexto, Holm, Lauridsen, Lund, Gronow, Niva e Mäkelä (2016) desenvolveram um estudo longitudinal com populações adultas em quatro países nórdicos (Noruega, Suécia, Dinamarca e Finlândia) nos anos de 1997 (n=4.823) e 2012 (n=8.242). O referido estudo ocorreu em três processos interligados que muitas vezes alegam ser distintivos da alimentação moderna: deslocação da alimentação de lares privados para ambientes comerciais, individualização sob a forma das pessoas comerem sozinhas e informalização, implicando em códigos de conduta mais casuais. 
Na obtenção dos resultados, foram comparados os dados sobre o local da refeição, com quem e por quanto tempo as pessoas levavam para comer e se ocorreram atividades paralelas durante a refeição. Os dados mostram que na localização primária (1997) das pessoas a alimentação continuava sendo o lar e o local de trabalho. Em 2012, a prática de comer sozinho aumentou ligeiramente na Dinamarca e na Noruega e diminuiu ligeiramente na Suécia. Além disso, houve maior propensão em comer às pressas, assistir televisão e o uso frequente de telas (tablets, computadores e smartphones), principalmente por pessoas mais jovens. Os autores concluíram que, mesmo enquanto as mudanças na organização social da alimentação não foram dramáticas, sinais de individualização e informalização puderam ser detectados (Holm et al., 2016).

Quanto as refeições em família, práticas alimentares e comensalidade, foram incluídos dois artigos realizados no Brasil. O primeiro artigo corresponde ao estudo transversal de base populacional, realizado por Sato, Lourenço, Trude, Unsain, Pereira, Martins, e Scagliusi (2016), que investigou as refeições em família e práticas alimentares entre 439 mães com 30 anos ou mais de idade na cidade de Santos. O referido estudo mostrou que a maioria das participantes $(83,6 \%)$ realizava refeições regulares com a família, das quais, aproximadamente 40,6\% compartilhavam pelo menos uma refeição, 28,0\% compartilhavam duas e $15,0 \%$ compartilhavam todas as refeições. O café da manhã foi consumido pela família em $25,5 \%$ dos participantes, enquanto 46,5\% e 69,7\%, respectivamente, almoçavam e jantavam com a família.

Apesar do percentual bastante expressivo de mães que realizam refeições em família $(83,6 \%)$, somente uma pequena parcela $(15,0 \%)$ fazia todas as refeições, o que pode ser atribuído ao estilo de vida e modernidade, no qual as mães saem de casa para trabalhar fora, o que limita as refeições em família, comensalidade e práticas alimentares. Quanto ao tipo de refeição, o jantar foi mais compartilhado por essas mães com suas famílias, o que deve coincidir com a volta para casa após jornada de trabalho no período da manhã e tarde. Por outro lado, o café da manhã foi a refeição com menor compartilhamento entre os familiares, podendo estar relacionado a saída de casa muito cedo para ir trabalhar (Sato et al., 2016).

O segundo artigo (Scagliusi, Pereira, Unsain \& Sato 2016), objetivou-se investigar as refeições familiares entre as mães $(n=30)$ que trabalhavam em universidades públicas brasileiras, e as associações entre comer com a família, dados sociodemográficos e práticas alimentares; avaliando a comensalidade em diversos ambientes do lar, seja à mesa, no sofá ou na cama. A mesa foi apontada como o local mais formal e valorizado para refeições em família, sendo o ambiente mais propenso à interação familiar e que sofre menor interferência da televisão, estando também relacionada à hierarquia, figuras de autoridade e modos à mesa. O sofá, no entanto, foi avaliado como um ambiente mais casual e descontraído, sofrendo grande interferência de telas, o que afeta a reunião familiar em torno de comer. Comer na cama é o local preferido para intimidades, embora possa também estar associado à precariedade, ou à solidão e separação, pois, na maioria das vezes, as pessoas apenas assistem televisão.

Destaca-se, também, a relação entre alimento, cultura e comensalidade. Nesse enfoque, uma pesquisa de caráter quantitativo e qualitativo com imigrantes indianos ( $\mathrm{n}=30$ ), com faixa etária de 25 a 50 anos e que residiam na Holanda entre 2 a 15 anos, avaliou as percepções sobre alimentação e identidade, práticas alimentares, culinária e práticas de comensalidade, na qual associou alimento/cultura e comensalidade à identidade, memórias, afeto, cuidado e bem-estar de um povo. A comensalidade entre indianos é uma maneira de restabelecer identidade e reafirmar a sua origem. Foi observado que praticamente não havia comensalidade entre indianos e holandeses, pois as práticas alimentares eram muito diferentes. Nesse sentido, observa-se o alimento como agente de exclusão (Bailey, 2017). Ademais, vale ressaltar que a escolha alimentar está ligada a características pessoais, da sociedade e da cultura das populações (Guine, Bartkiene, Szucs, Tarcea, Ljubicic, Cernelic-Bizjak, Isoldi, El-Kenawy, Ferreira, Straumite, Korzeniowska, Vittadini, Leal, Frez-Munoz, Papageorgiou, Djekic, Ferreira, Correia, Cardoso \& Duarte, 2020).

No tocante as refeições em família e bem-estar, tem-se o estudo transversal de Utter, Larson, Berge, Eisenberg, Fulkerson e Neumark-Sztainer (2018), com banco de dados de pesquisas de base populacional, no qual foi efetivado com uma 
amostra de pais nos Estados Unidos da América ( $\mathrm{n}=889$, idade média de 31 anos), que responderam à quarta onda do estudo Projeto Alimentação e Atividade em Adolescentes e Jovens Adultos (EAT) dos 1.830 participantes, $49 \%(\mathrm{n}=889)$ relataram ter pelo menos um filho e foram selecionados nessa pesquisa.

Os relatos de refeições familiares entre pais e filhos foram associados a níveis mais altos de interação familiar, maior autoestima e níveis mais baixos de sintomas depressivos e estresse. A frequência das refeições em família também esteve relacionada ao maior consumo de frutas e vegetais. As associações entre a frequência das refeições familiares e o bem-estar dos pais foram semelhantes tanto para mães como para pais. Os achados do presente estudo sugerem que refeições familiares frequentes podem contribuir para o bem-estar social e emocional dos pais. Nesse sentido, esses achados são significativos, pois é bem conhecido que o bem-estar mental dos pais afeta a saúde e o bem-estar de seus filhos (Utter et al.,2018).

Considerando-se todo o exposto, ressalta-se que a vida moderna tem ocasionado mudanças socioeconômicas e demográficas que proporcionaram alterações no modo de vida dos indivíduos, como modificação no comportamento alimentar (World Health Organization, 2004). Em adição, é importante destacar que a globalização alimentar se deu a partir da ruptura radical dos sistemas alimentares, ou seja, das escolhas e práticas de cozinha de uma região, repercutindo na interação com produtos de todo o mundo (Maluf, Menezes \& Valente, 1996), tendo em vista, a livre circulação de capital, de tecnologia, bens e serviços, com efeitos profundos nos estilos de vida ligados à dieta (Popkin, 2006), pois diferentes povos são aptos a novos sabores, sentidos, hábitos, que se definem desde o plantio até o consumo (Pineyrua, 2006).

O guia alimentar brasileiro, por sua vez, traz como uma das recomendações básicas, o compartilhamento de refeições, que pode influenciar no consumo alimentar, no convívio com os familiares e no prazer proporcionado pela alimentação (Brasil, 2014). Além disso, o ato de comer em companhia favorece o convívio social e interações que atuam como referência de cultura, demonstração de afeto, compromisso e identidade (Busato, Pedrolo, Gallina \& Rosa, 2015; Cardoso, Santo, Nunes \& Loureiro, 2015). Entretanto, a comensalidade pode também ser percebida como algo negativo, que gera tensões, dependendo do contexto, como também das características das pessoas envolvidas nesse compartilhamento (Giacoman, 2016).

O comer em companhia pode ser dificultado por diversos fatores, como escassez de tempo para o preparo e consumo alimentar, deslocamento das refeições e aumento da individualização dos rituais que envolvem a alimentação. Isso contribui para que, também, ocorra modificação no padrão alimentar que passa a ser representado por produtos industrializados e prontos para o consumo, como os produtos ultraprocessados, pois possuem elevada densidade calórica, são ricos em gorduras, açúcares, colesterol e sódio, constituindo-se como uma preocupação para a saúde dos indivíduos, haja vista a relação existente entre o consumo inadequado de alimentos e o desenvolvimento de Doenças Crônicas Não Transmissíveis (DCNT) (Brasil, 2014; World Health Organization, 2018).

Apesar dos obstáculos, a realização e o compartilhamento de refeições em família, ainda era presente nas famílias cujas mães eram responsáveis em produzir a alimentação e, entre as mães que consumiam quantidade menor de alimentos, em relação aos outros familiares (Sato et al., 2016). Isso pode sinalizar a importância da mulher, que embora tenha ingressado no mercado de trabalho, ainda cabe a mesma a responsabilidade pela produção e pelo compartilhamento das refeições na família (Brasil, 2008).

No Brasil, o estudo realizado por Andrade, Gombi-Vaca, Louzada, Azeredo e Levy, (2020), a partir de dados da Pesquisa de Orçamento Familiar (POF) 2008-2009, do Instituto brasileiro de Geografia e Estatística, revelou associação negativa entre o consumo de alimentos ultraprocessados e a contribuição de nutrientes (proteínas, vitaminas, minerais e fibras) e associação positiva em relação ao aporte de açúcar livre, e gorduras (totais, saturadas e trans). Além disso, foi constatado maior consumo de alimentos ultraprocessados, quando as refeições eram realizadas fora do domicílio.

Nesse contexto, Bezerra, Moreira, Cavalcante, Souza e Sichieri (2017) referem que alimentação fora do domicílio pode ser um marcador de risco para práticas alimentares pouco saudáveis. Tal fato é preocupante, uma vez que, a realização de 
refeições fora da residência é uma tendência mundial, decorrente da globalização, urbanização, dos avanços tecnológicos na cadeia produtiva de alimentos, questões de caráter demográfico, socioeconômico e cultural e mudanças no estilo de vida das pessoas (Luizetto et al., 2015).

Segundo a POF 2017-2018 o gasto com a alimentação fora do domicílio, como por exemplo em restaurantes e fastfoods, passou de 24,1\% para 32,8\% entre os anos 2002 e 2018, com aquisição de alimentos não saudáveis. Outro dado, preocupante, é a mudança de comportamento alimentar, no qual foi verificado em nível domiciliar, com aumento de bebidas (refrigerantes e sucos industrializados) e alimentos prontos para o consumo (Instituto Brasileiro de Geografia e estatística, 2020).

De acordo com as diretrizes alimentares brasileiras, os produtos ultraprocessados apresentam elevada densidade calórica, contribuem para o aporte calórico excessivo, interferem negativamente nos mecanismos de controle e a saciedade e, devido ao sabor acentuado, contribuem para o consumo involuntário. Além disso, possuem facilidade de serem consumidos em qualquer lugar, sem utilização de utensílios, e diante de telas, para o comer rápido e sem atenção. Logo, o consumo desse tipo de alimento colabora para o aumento do risco de obesidade e de outros tipos de DCNT (Brasil, 2014).

Esse comportamento, decorrente da vida moderna, de realizar de refeições na presença de telas, dentre outros, foi evidenciado na presente revisão, por meio das análises dos estudos de Scagliusi et al., (2016), onde a família tinha o hábito de consumir alimento assistindo televisão, seja na mesa, no sofa ou na cama, Holm et al., (2016), onde houve aumento de comer diante de telas, principalmente por pessoas mais jovens, conforme já mencionado anteriormente.

Ressalta-se que, globalmente, os ultraprocessados podem ser similares quanto aos conteúdos, rótulos e outras características. As marcas mais conhecidas são propagadas frequentemente em grandes campanhas públicitárias., que visam a promoção do desejo de consumir cada vez mais esses produtos e da sensação de pertencimento dos consumidores a uma cultura moderna. Isso traz como consequências, o desinteresse no consumo de alimentos tradicionais, que fazem parte de culturas alimentares genuínas (Brasil, 2014).

Esse desinteresse foi constatado na presente revisão, onde houve maior valorização de novos alimentos, pertencentes a outras culturas por mães latinas (Roche et al., 2015). A desvalorização da cultura alimentar tradicional, decorrente da vida moderna, pode levar a perda da identidade (Brasil, 2008). De outro modo, foram identificados a preservação da cultura e identidade alimentar entre migrantes indianos, pois trouxeram consigo os alimentos e práticas alimentares que fazem parte de sua cultura nativa (Bailey, 2017), e a valorização dos alimentos tradicionais por um grupo étnico (hmong) (Roche et al., 2015).

Assim, a globalização para além de discussões ideológicas é um dos processos de aprofundamento da integração econômica, social, cultural e política que vem impulsionando o mundo, considerando, principalmente, as facilidades de transporte e difusão de informações, por meios dos meios de comunicação, produtos e interação entre as pessoas (Proença, 2010).

\section{Conclusão}

O comportamento alimentar sofre interferência diante da modernidade e da globalização. Observa-se mudanças drásticas nos hábitos e práticas alimentares, com tendência a piora da qualidade das refeições, diminuição da socialização e compartilhamento de refeições entre familiares, difusão e fragmentação cultural. $O$ ato de comer com prazer pode ser comprometido, pela pressa da rotina diária, distância de casa, ausência de interação social ou por falta de opções de alimentos no mercado com os quais os indivíduos se identifiquem próprios da sua cultura.

Existe uma inclinação à supervalorização da cultura alimentar de origem e a tentativa de sua reprodução, principalmente quando se convive com culturas diferentes, dentro das quais a culinária pode ser um agente de exclusão social, sendo o resgate da cultura de origem uma maneira de se reafirmar como indivíduo. 
O uso de telas influencia negativamente na comensalidade, na interação e diálogo entre as pessoas e no local das refeições, pois predispõe o deslocamento da sala de jantar para locais onde é comum a presença de televisão, que ao distrair o comensal, contribui para maior consumo alimentar, elevando o risco de desenvolvimento de obesidade. Nesse contexto, as perspectivas são preocupantes, pois se espera uma diminuição ainda maior na interação social durante as refeições. Assim, ressalta-se a importância da realização de novos estudos em relação a temática trabalhada, de forma que contribuam para obtenção de estratégias que provoquem modificações nesse cenário.

\section{Referências}

Alvarenga, M. S., Figueiredo, M., Timerman, F., \& Antonaccio, C. M. A. (2019). Nutrição comportamental: Manole.

Andrade, G. C., Gombi-Vaca, M. F., Louzada, M. L. C., Azeredo, C. M., \& Levy, R. B. (2020). The consumption of ultra-processed foods according to eating out occasions. Public Health Nutrition, 23(6), 1041-1048.

Azevedo, A. (2017). Alimentação, sociedade e cultura: temas contemporâneos. Sociologias, 9(44), 276-307.

Bailey, A. (2017). The migrant suitcase: Food, belonging and commensality among Indian migrants in The Netherlands. Appetite, 1(110), 51-60.

Bezerra, I. N., Moreira, T. M. V., Cavalcante, J. B., Souza, A. M., \& Sichieri, R. (2017). Consumo de alimentos fora do lar no Brasil segundo locais de aquisição. Revista de Saúde Pública, 51, 1-8.

Busato, M. A., Pedrolo, C., Gallina, L. S., \& Rosa, L. (2015). Ambiente e alimentação saudável: percepções e práticas de estudantes universitários. Semina: Ciências Biológicas e da Saúde, 36(2), 75-84.

Cardoso, S., Santo O., Nunes, C., \& Loureiro, C. (20015). Escolhas e hábitos alimentares em adolescentes: associação com padrões alimentares do agregado familiar. Revista Portuguesa de Saúde Pública, 33(2), 128-136.

Carneiro, H. (2017). Comida e sociedade: uma história da alimentação. Elsevier Brasil.

Carvalho, L. G. A., Bastos, S. R., \& Gimenes-Minasse, M. H.S. G. (2017). Comensalidade na Família Nuclear Paulistana: 1950 a 2000. Rosa dos VentosTurismo e Hospitalidade, 9(1),18-31.

Cho, W., Takeda, W., Oh, Y., Aiba, N., \& Lee, Y. (2015). Perceptions and practices of commensality and solo-eating among Korean and Japonese university students: A cross-cultural analysis. Nutrition Research and Practice, 9(5), 523-9.

Giacoman, C. (2016). The dimensions and role of commensality: A theoretical model drawn from the signicance of communal eating among adults in Santiago, Chile. Appetite, 1(107), 460-470.

Guillemi, I., Marrel, A., Arnould, B., Capuron, L., Dupuy, A., Ginon, E., Layé, S., Lecerf, J. M., Prost, M. Rogeaux, M., Urdapilleta, I., \& Allaert, F. A. (2016). How French subjects describe well-being from food and eating habits? Development, item reduction and scoring definition of the Well-Being related to Food Questionnaire (Well-BFQ@). Appetite, 96, 333-346.

Guine, R. P. F., Bartkiene, E., Szucs, V., Tarcea, M., Ljubicic, M., Cernelic-Bizjak, M., Isoldi, K., El-Kenawy, A., Ferreira, V., Straumite, E., Korzeniowska, M., Vittadini, E., Leal, M., Frez-Munoz, L., Papageorgiou, M., Djekic, I., Ferreira, M., Correia, P., Cardoso, A. P., \& Duarte, J. (2020). Study about Food Choice Determinants According to Six Types of Conditioning Motivations in a Sample of 11,960 Participants. Foods, 9(7), 888-906.

Holm, L., Lauridsen, D., Lund, T. B., Gronow, J., Niva, M., \& Mäkelä, J. (2016). Changes in the social context and conduct of eating in four Nordic countries between 1997 and 2012. Appetite, 1(103), 358-368.

Hooper, C. M., Ivory, V. C., \& Fougere, G. (2015). "Dinner's ready!” A qualitative exploration of the food domain across the lifecourse. Appetite, 92,133-142.

Instituto Brasileiro de Geografia e Estatística. (2020), Pesquisa de orçamentos familiares 2017-2018: avaliação nutricional da disponibilidade domiciliar de alimentos no Brasil. Rio de Janeiro (RJ): IBGE.

Lima, R. S., Ferreira Neto, J. A., \& Farias, R. C. P. (2015). Alimentação, comida e cultura: o exercício da comensalidade. Demetra: Alimentação, Nutrição \& Saúde, 10(3), 507-522.

Luizetto, E. M., Tureck, C., Locatelly, G., Correa, V. G., \& Koehnlein, E. A. (2015). Alimentos Funcionais em Alimentação Coletiva: refeições acerca da promoção da saúde fora do domicílio. Nutrire, 40(2), 188-199.

Maluf, R. S., Menezes, F., \& Valente, F. L. (1996). Contribuição ao tema da segurança alimentar no Brasil. Cadernos de Debate, 4(1), 66-88.

Ministério da Saúde. Secretaria de Atenção à Saúde. (2014). Guia alimentar para a população brasileira. (2a ed.,) Ministério da Saúde.

Ministério da Saúde. Secretaria de Atenção à Saúde. (2008), Guia alimentar para a população brasileira: promovendo a alimentação saudável. (1ª ed.,): Ministério da Saúde, 2008.

Pineyrua, D. G. F. (2006). Regionalizmo alimentar: identificação de grupos de consumidores que valorizam o prazer e as tradições alimentares. Dissertação (Mestrado em Agronegócios) - Universidade Federal de Goiás, Goiânia. 
Research, Society and Development, v. 10, n. 5, e26710514242, 2021

(CC BY 4.0) | ISSN 2525-3409 | DOI: http://dx.doi.org/10.33448/rsd-v10i5.14242

Popkin, B. M. (2006). Global nutrition dynamics: the world is shifting rapidly toward a diet linked with noncommunicable diseases. American Journal of Clinical Nutrition, 84(1), 289-298.

Proença, R. P. da C. (2010). Alimentação e globalização: algumas reflexões. Ciência e Cultura, 62(4), $43-47$.

Proença, R. P. C. (2010). Alimentação e globalização: algumas reflexões. Ciência e Cultura, 62(4), 43-47.

Roche, A., Goto, K., Zhao, Y., \& Wolff, C. (2015). Bonding and Bridging Social and Cultural Capitals: Perceived Factors Associated with Family Eating Practices Among Hmong, Latino, and White Mothers and Fathers. Journal of Nutrition Education and Behavior, 47(6), 540-547.

Santos, P. H. R., \& Sousa, P. H. M. (2021). Food memories of the residents of the Municipality of Beberibe - Ceará: a report on the food cultural manifest. Research, Society and Development, 9(8), e748986527.

Sato, P. M., Lourenço, B. H., Trude, A. C. B., Unsain, R. F., Pereira, P. R., Martins, P. A., \& Scagliusi, F. B. (2016). Family meals and eating practices among mothers in Santos, Brazil: a population-based study. Appetite, $1(103), 38-44$.

Scagliusi, F. B., Pereira, P. R., Unsain, R. F., \& Sato P. (2016). Eating at the table, on the couch and in bed: An exploration of different locus of commensality in the discourses of Brazilian working mothers. Appetite, 1(103), 80-86.

Utter, J., Larson, N., Berge, J. M., Eisenberg, M. E., Fulkerson, J. A., \& Neumark-Sztainer, D. (2018). Family meals among parents: Associations with nutritional, social and emotional wellbeing. Preventive Medicine, 113, 7-12.

World Health Organization. (2004). Global strategy on diet, physical activity and health.

World Health Organization. (2018). Obesity and overweight.

Zaneti, T. B. (2016). A cozinha gaúcha: um resgate dos sabores e saberes da gastronomia do Rio Grande do Sul. Ágora, 18(1), 28-42. 